\title{
Real-Time and In Situ Evaluation of Phycocyanin Concentration in Spirulina platensis Cultivation System by Using Portable Raman Spectroscopy
}

\author{
Yong He, ${ }^{1,2}$ Xiaoxi Liu, ${ }^{1,2}$ Hui Fang, ${ }^{1,2}$ Jinnuo Zhang, ${ }^{1,2}$ and Xuping Feng $\mathbb{D}^{1,2}$ \\ ${ }^{1}$ College of Biosystems Engineering and Food Science, Zhejiang University, Hangzhou 310058, China \\ ${ }^{2}$ State Key Laboratory of Modern Optical Instruments, Zhejiang University, Hangzhou 310027, China \\ Correspondence should be addressed to Xuping Feng; fengxp@zju.edu.cn
}

Received 15 September 2020; Revised 17 February 2021; Accepted 24 February 2021; Published 2 March 2021

Academic Editor: Jean-Marie Nedelec

Copyright ( $\odot 2021$ Yong He et al. This is an open access article distributed under the Creative Commons Attribution License, which permits unrestricted use, distribution, and reproduction in any medium, provided the original work is properly cited.

Spirulina platensis can synthesize a large amount of phycocyanin, which had been developed as a health food. At the same time, Spirulina can absorb the nitrogen and phosphorus in wastewater and provide for its own growth. Here, we studied the optimal nitrogen and phosphorus supply for the Spirulina production process. For the first time, $405 \mathrm{~nm}$ portable Raman spectrometer was used to estimate phycocyanin content for real-time industrial applications. We obtained three Raman characteristic peaks of phycocyanin through density functional theory combined with home-built Raman spectrometer, which were 1272, 1337, and $1432 \mathrm{~cm}^{-1}$. There was a good linear correlation between the sum of the three peak intensities and the PCL concentration $\left(y=18.887 x+833.530, R^{2}=0.890\right)$. The least squares support vector machine model based on the characteristic peaks was used to estimate the concentration of phycocyanin and obtained good results with a correlation coefficient of prediction of 0.907 and residual predictive deviation of 3.357. The results can provide decision-making for integration of Spirulina effluent treatment and phycocyanin production and provide references for real-time Spirulina-based biorefinery applications.

\section{Introduction}

With the development of industry and agriculture, the living standard has been continuously improved. However, it has also produced a lot of wastewater, which has caused many pollution problems. Due to the large amount of N and P in the wastewater, the problem of eutrophication appears, which needs to be effectively controlled and solved. Algae have strong heterotrophic and polytrophic capabilities, which can recycle nutrients in wastewater and produce high value-added products. It is therefore considered to be ideal biomaterials for the comprehensive utilization of wastewater. Since this century, the research focused on wastewater treatment accounted for $25 \%$ of the algae-related research [1]. Gao et al. [2] used a membrane photobioreactor to develop a highly efficient algae cultivation process for removing nutrients from wastewater. The treatment was generally to use the organic substances in the wastewater to support growth for algae and then achieve the purpose of purifying the wastewater. During the growth of algae, many valuable products will be produced, such as phycocyanin, lipids, and pigment [3]. Spirulina platensis can synthesize phycocyanin and can be used for photosynthesis and heterotrophic cultivation [4]. As a rare water-soluble blue pigment-protein complex, phycocyanin had wide application prospects in beverage coloring, health food, biotechnology, and other industries [5]. Wang et al. [6] used livestock and poultry farming wastewater as a medium to cultivate $S$. platensis. The results showed it not only had rich biomass but also can remove the nitrogen $(\mathrm{N})$ and phosphorus $(\mathrm{P})$ in wastewater effectively. However, it will cause stress on the growth of S. platensis when the concentration of $\mathrm{N}$ and $\mathrm{P}$ was not suitable $[7,8]$. Therefore, it is particularly important to find a suitable concentration of $\mathrm{N}$ and $\mathrm{P}$ that is conducive to the accumulation of the phycocyanin. In this production process, dynamic and real-time monitoring of phycocyanin content is very necessary. As commonly detection methods, spectrophotometry and fluorophotometer always have good accuracy but are of high cost [9]. Moreover, these methods require complicated and destructive 
preprocessing, which are tedious and time-consuming. Besides, the temperature and storage time will affect the stability of phycocyanin. Therefore, the development of rapid nondestructive methods for the detection of phycocyanin is very significant for the development of dynamic cultivation strategies. The Raman technology was widely used in the detection of biomacromolecules in microalgae, such as pigments $[10,11]$, proteins [12], lipids [13, 14], and the mechanism of intracellular biochemical reactions $[15,16]$. The advantages of very weak influence from aqueous solution and no pretreatment make it possible for rapid in situ determination of phycocyanin during the cultivation process. Jehlicka and Oren [17] used a handheld Raman spectrometer to detect phycocyanin in cyanobacteria. Ando et al. [18] used micro-Raman spectroscopy to obtain high signal-to-noise ratio spectra of phycocyanin in a single living cyanobacteria cell. At present, the detection of phycocyanin by Raman spectroscopy was mainly focused on qualitative aspects, but there were few studies on the quantitative detection of phycocyanin. With the gradual expansion market of phycocyanin, convenient and rapid quantitative monitoring methods are needed to guide industrial production. The main analytical procedure of this study is displayed in Figure 1, which include the following: (1) to find the most suitable concentration of $\mathrm{N}$ and $\mathrm{P}$ that can support the growth of Spirulina to achieve the dual effects of wastewater purification and cost saving, (2) to find the Raman characteristic peak of phycocyanin, and (3) to achieve in situ rapid detection of phycocyanin by Raman spectroscopy.

\section{Materials and Methods}

2.1. Algae Species and Culture Conditions. Spirulina platensis (FACHB-882) was purchased from the Wildlife Germplasm Bank of Chinese Academy of Sciences. Spirulina was cultivated in a triangular flask and expanded for cultivation using the Spirulina medium (SP medium) [19] under controlled conditions $\left(25^{\circ} \mathrm{C}, 8000 \mu \mathrm{mol}\right.$ (photon), $12 \mathrm{~h}$ day). According to the previous analysis, wastewater containing $\mathrm{N}$ and $\mathrm{P}$ could be used to cultivate Spirulina to accumulate phycocyanin. The experimentation was first carried out to investigate the appropriate $\mathrm{N}$ and $\mathrm{P}$ supply for $\mathrm{S}$. platensis cultivation. Chemical reagent $\mathrm{NH}_{4} \mathrm{Cl}$ was supplied as the $\mathrm{N}$ source, while $\mathrm{K}_{2} \mathrm{HPO}_{4}$ was supplied as the $\mathrm{P}$ source. A blend of three $\mathrm{N}$ treatment was used along with three different $\mathrm{P}$ levels, and the formulation of these grades is presented in Table 1. Samples that were cultivated using the SP medium were regarded to be the control group, in which the concentration of $\mathrm{NaNO}_{3}$ was $2.5 \mathrm{~g} / \mathrm{L}$ and the $\mathrm{K}_{2} \mathrm{HPO}_{4}$ was $0.5 \mathrm{~g} / \mathrm{L}$. During the experiment, dry cell weight (DCW), phycocyanin content in liquid (PCL), and phycocyanin content in dried Spirulina (PCD) were measured $0,4,7,11,14$, and 18 days after treatment.

2.2. Biochemical and Physiological Parameter Measurements. DCW, a proxy of Spirulina biomass, was determined by the gravimetric method. The algal muds of each sample were wrapped in tin foil tray and dried at $40^{\circ} \mathrm{C}$ to stable weights. The DCW was calculated as

$$
\mathrm{DCW}=\frac{\left(m_{1}-m_{0}\right)}{V},
$$

where DCW was the dry cell weight of Spirulina $(\mathrm{g} / \mathrm{L}), m_{1}(\mathrm{~g})$ was the weight of the foil tray $(\mathrm{g}), m_{0}(\mathrm{~g})$ was the total weight of tin foil tray and dried algae mud, and $V$ was the volume of algal fluid $(L)$.

Transfer the dried Spirulina to a centrifuge tube, and add liquid nitrogen to freeze the cells in order to make the cell walls more fragile. After complete evaporation of the liquid nitrogen, the wall began to grind and crack. It was then shaken to dissolve completely the phycocyanin, the mixture was centrifuged at $8000 \mathrm{r} / \mathrm{min}$, and take the supernatant as the detection solution. The phycocyanin was detected by a dual-beam ultraviolet spectrophotometer (TU-1901, Beijing General Analysis, China). There were two absorption peaks at 348 and $620 \mathrm{~nm}$ (Figure 2(a)), which was consistent with the result in some research studies [20]. The phycocyanin standard curve (Figure 2(b)) was obtained at $620 \mathrm{~nm}$. The content of phycocyanin in liquid (PCL) was calculated as

$$
\text { PCL }=\left(406.479 A_{620}-0.213\right) \frac{V_{1}}{V},
$$

where PCL was the content of phycocyanin in liquid $(\mathrm{mg} / \mathrm{L})$, $A_{620}$ was the absorbance value at $620 \mathrm{~nm}, V_{1}$ was the volume of phosphate buffer (L), and $V$ was the volume of Spirulina fluid $(L)$.

The phycocyanin content in dried Spirulina (PCD) was calculated as

$$
\mathrm{PCD}=\frac{\mathrm{PCL}}{\mathrm{DCW}}
$$

where PCD was the phycocyanin in dry algae $(\mathrm{mg} / \mathrm{g}), \mathrm{PCL}$ was the content of phycocyanin in liquid $(\mathrm{mg} / \mathrm{L})$, and DCW was the dry cell weight of Spirulina $(\mathrm{g} / \mathrm{L})$.

2.3. Raman Spectrum Acquisition. The portable Raman spectrometer in this study was designed and built by our own institute. The devices of this system (Figure 3 ) include a $405 \mathrm{~nm}$ Raman probe, a Raman spectrum detector, a liquid sample cell with blackout cover, a laptop with XRaman software, an optical fiber, and a shading box. Calibrate the instrument by a $405 \mathrm{~nm}$ excitation wavelength before spectrum acquisition. The parameters were set as follows: a scanning range of 151 to $3000 \mathrm{~cm}^{-1}$, an integration time of $8000 \mathrm{~ms}$, and an average spectral value of twice. When collecting the spectrum of samples, $1.5 \mathrm{ml}$ of Spirulina liquid was added into a $2 \mathrm{~mL}$ quartz bottle and then placed at a liquid sample pool. Since the spectrum may be affected by noise and fluorescence, we measured 4 replicates for each sample. 96 spectral data were obtained, and it was divided into a calibration set of 64 individuals and a prediction set of 32 individuals, before constructing the prediction model. 


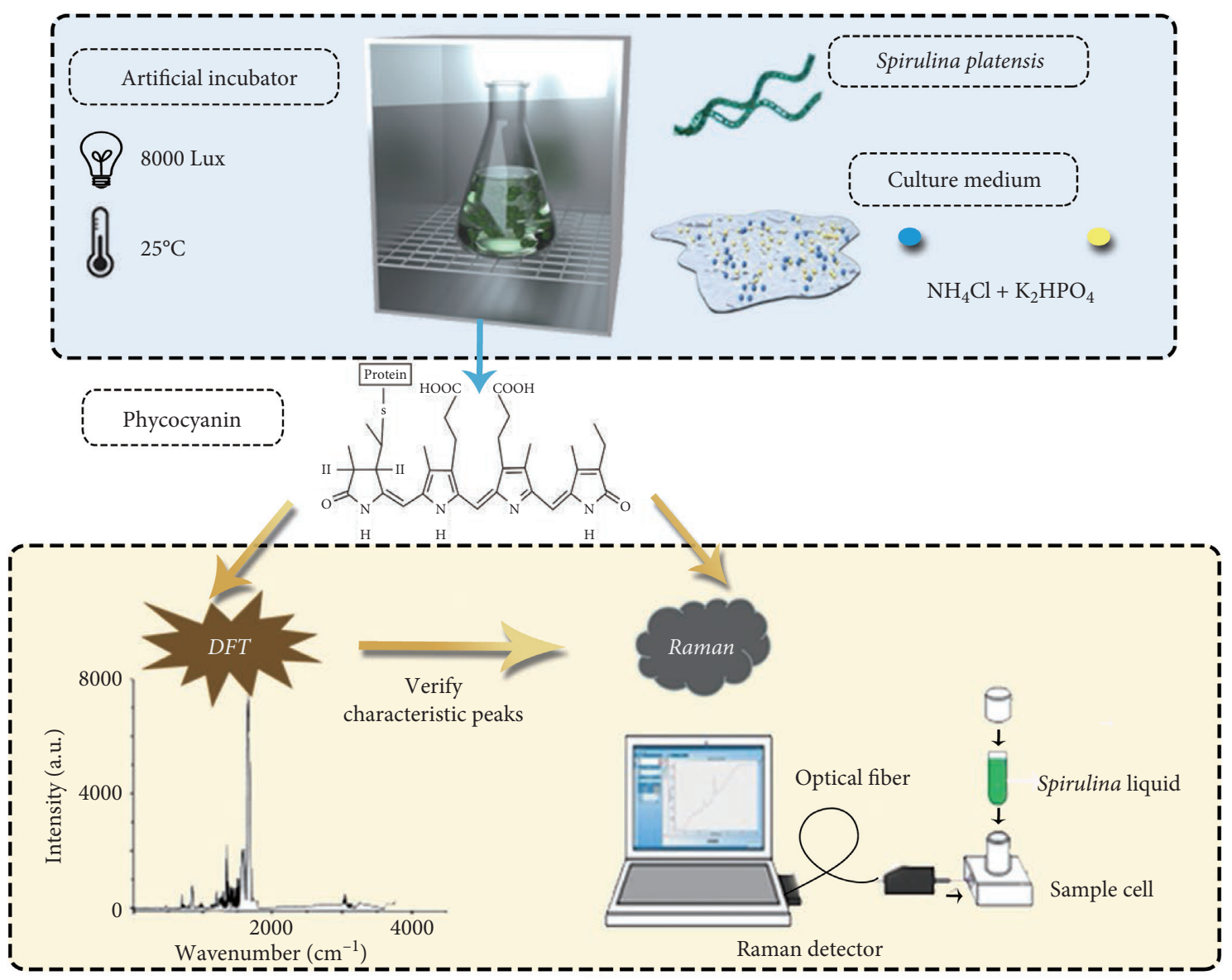

Figure 1: Schematic overview of the analytical procedure.

TABLE 1: Concentration of culture medium components in the experimental group.

\begin{tabular}{lcc}
\hline Group & $\mathrm{NH}_{4} \mathrm{Cl}(\mathrm{g} / \mathrm{L})$ & $\mathrm{K}_{2} \mathrm{HPO}_{4}(\mathrm{~g} / \mathrm{L})$ \\
\hline N1P1 & 0.1 & 0.02 \\
N1P2 & 0.1 & 0.42 \\
N1P3 & 0.1 & 0.82 \\
N2P1 & 0.2 & 0.02 \\
N2P2 & 0.2 & 0.42 \\
N2P3 & 0.2 & 0.82 \\
N3P1 & 0.3 & 0.02 \\
N3P2 & 0.3 & 0.42 \\
N3P3 & 0.3 & 0.82 \\
\hline
\end{tabular}

\subsection{Analytical Methods}

2.4.1. Density Functional Theory. Density functional theory (DFT) was a quantum mechanical method used to study the electronic structure of multielectron systems with varieties of calculation functions and basis sets [21]. In order to explain mechanism behind the formation of these absorption peaks, quantum chemistry calculations of the phycocyanin chromophore molecule were carried out using Gaussian.v09 (Gaussian, Inc., Wallingford, CT, USA). The hybrid functional model of B3LYP with the 6$311 \mathrm{G}$ basis set was used to optimize the single molecular structure of phycocyanin and simulate its DFT spectrum. The molecular vibration modes of each peak were determined by comparing the experimental and theoretical spectra.

2.4.2. Predictive Model. In this step, least squares support vector machine (LSSVM) was used to establish a quantitative detection model of phycocyanin. LSSVM is a regression method based on support vector machine (SVM) algorithm. It has certain applications in qualitative and quantitative analyses of spectra [22]. The LSSVM model transforms 


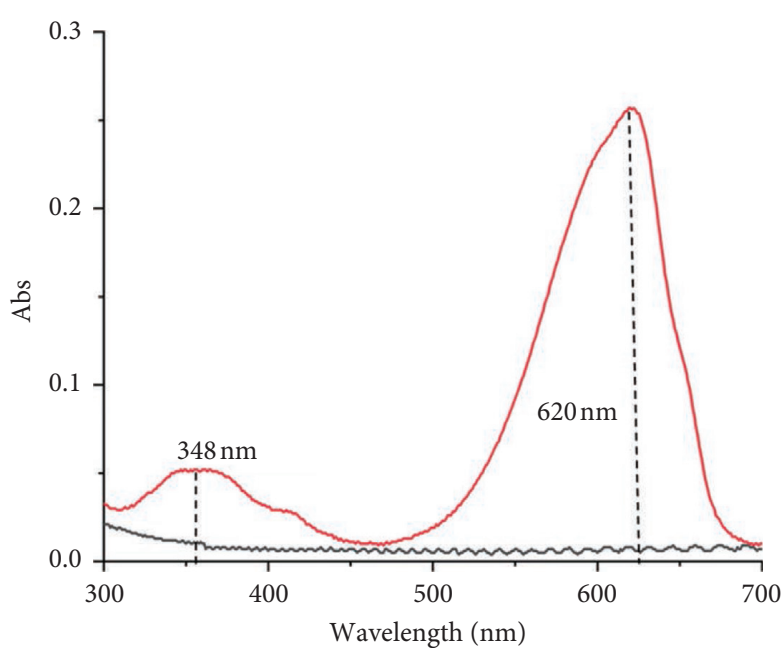

- $\mathrm{PB}$

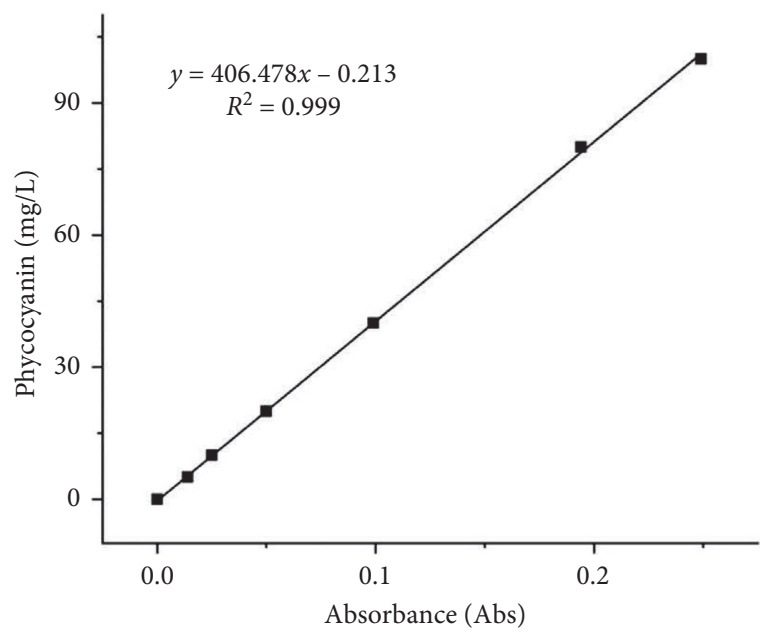

(b)

Figure 2: (a) Absorbance spectrum of phycocyanin (PC) standard solution and its solvent (PB); (b) standard curve for the absorbance of phycocyanin.

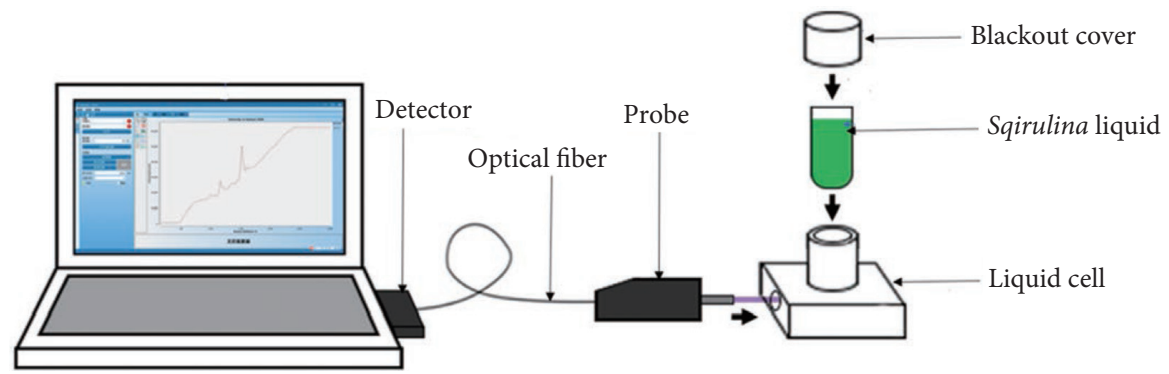

FIGURE 3: Detection process of the portable Raman spectrometer.

inequality constraints of SVM into equality constraints, which help to reduce the problem to solve linear equations. The LSSVM model was operated with the redial basis function as a kernel function. The $\operatorname{sig}^{2}\left(\sigma^{2}\right)$ and gam $(\gamma)$ were determined based on the grid search technique. The $\sigma^{2}$ represents the bandwidth of redial basis function kernel. $\gamma$ represented the tradeoff between minimizing model complexity and minimizing the training error.

2.5. Evaluation of Model Performance. The performance of LSSVM model was evaluated by calculating the coefficients of determination in calibration sets $\left(R_{\text {cal }}^{2}\right)$ and prediction sets $\left(R_{\text {pre }}^{2}\right)$, as well as root mean square error in calibration sets (RMSEC) and prediction sets (RMSEP). The metric residual predictive deviation (RPD) expresses the ratio of the standard deviation of the data set and RMSEP, which can evaluate the predictive ability of the model. It is generally believed that when $1.75<\mathrm{RPD}<2.25$, the model is valid; when $2.25<\mathrm{RPD}<3$, the model is more successful; $3<\mathrm{RPD}<4$, the model is successful; when $\mathrm{RPD}>4$, the model is very successful. Therefore, the larger the RPD value is, the better the model is.
2.6. Software. Matlab ${ }^{\circledR} 2018 b$ (The Math-Works, Natick, MA, USA) was used for regression model establishment and calculation of model evaluation parameters. Gaussian.v09 (Gaussian, Inc., Wallingford, CT, USA) was used to do the DFT calculation. The SPSS ver.22 (SPSS Inc., Chicago, IL, USA) was used to analyze the biochemical and physiological parameters. Comparisons of response value of DCW, PCD, and PCL were made using ANOVA. The significance of variables was determined using the $\mathrm{F}$ test $(p<0.01)$.

\section{Results and Discussion}

\subsection{Environmental Impact of Spirulina Growth and Phycocyanin Accumulation}

3.1.1. Effects of Different $N$ and P Levels on Spirulina Growth and Phycocyanin Accumulation. DCW represented the biomass of Spirulina, and its trend over time is shown in Figure 4(a). In the control group and N1 group, the biomass of Spirulina increased significantly over time. However, with the increased $\mathrm{N}$ supply (N2 and N3), the DCW began to decrease. These results indicated that high $\mathrm{N}$ supply will 


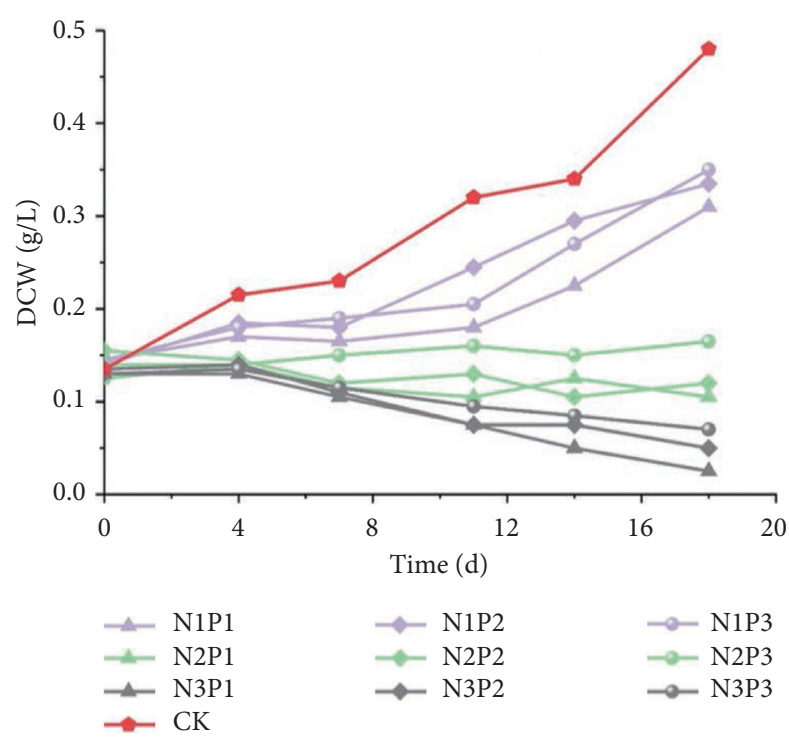

(a)

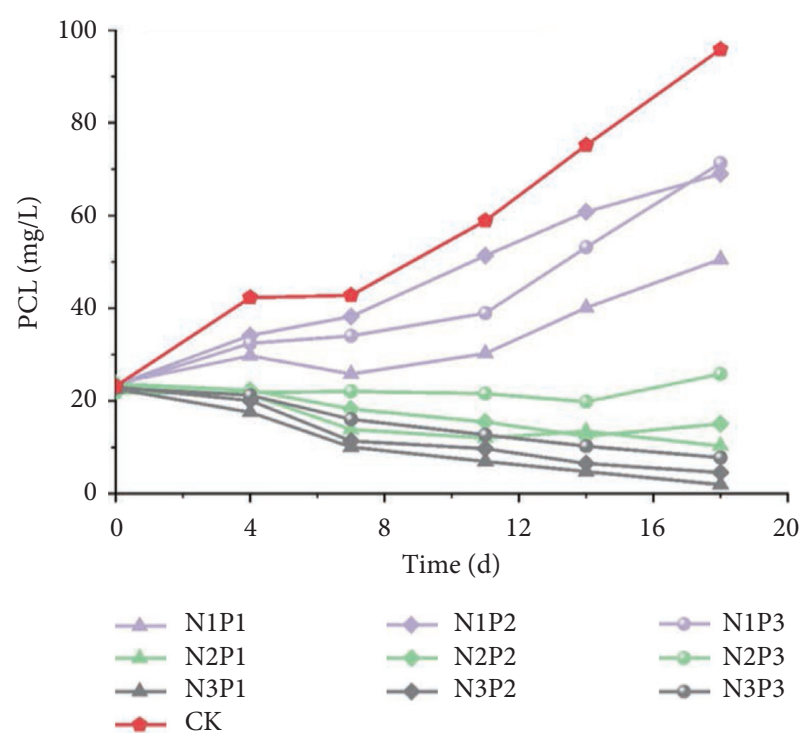

(b)

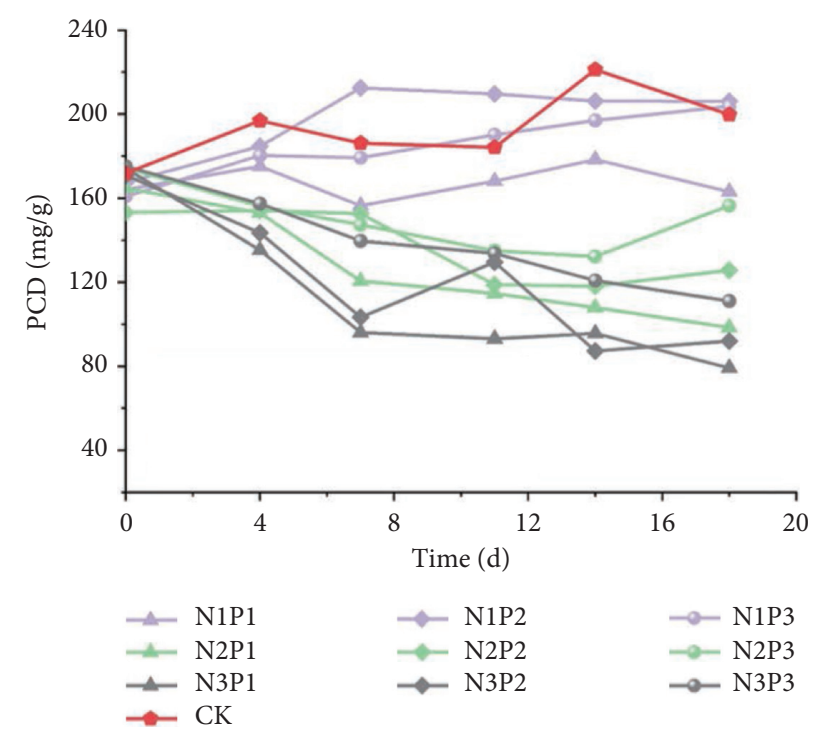

(c)

Figure 4: Change process of (a) dry cell weight (DCW), (b) the content of phycocyanin in liquid (PCL), and (c) the content of phycocyanin in dry Spirulina (PCD). The CK is the control group, using Spirulina medium with $2.5 \mathrm{~g} / \mathrm{L}$ concentration of $\mathrm{NaNO}_{3}$ and $0.5 \mathrm{~g} / \mathrm{L} \mathrm{K}_{2} \mathrm{HPO}_{4}$. The experimental group consists of different concentrations of $\mathrm{NH}_{4} \mathrm{Cl}(\mathrm{N} 1,0.1 \mathrm{~g} / \mathrm{L} ; \mathrm{N} 2,0.2 \mathrm{~g} / \mathrm{L} ; \mathrm{N} 3,0.3 \mathrm{~g} / \mathrm{L})$ and $\mathrm{K}_{2} \mathrm{HPO}_{4}(\mathrm{P} 1,0.02 \mathrm{~g} / \mathrm{L} ; \mathrm{P} 2$, $0.42 \mathrm{~g} / \mathrm{L} ; \mathrm{P} 3,0.82 \mathrm{~g} / \mathrm{L})$.

cause stress and hinder the growth of Spirulina [23]. In the N1 group, the biomass in N1P2 and N1P3 was higher than $\mathrm{N} 1 \mathrm{P} 1$. In the N2 group, the biomass in N2P3 had a certain increase, while the group with a lower concentration of $\mathrm{P}$ (N2P1 and N2P2) showed a downward trend. In the N3 group, the higher the concentration of the $\mathrm{P}$, the more gradual the downward trend. These results may indicate that when the Spirulina was damaged by high concentration of N, the high concentration of P can protect the Spirulina and then increase the biomass [24]. The overall trend of PCL (Figure 4(b)) was similar to DCW. When the concentration of $\mathrm{N}$ was low, the biomass tended to increase. At higher $\mathrm{N}$ concentrations, the PCL was gradually decreasing. The trend of PCD (Figure 4(c)) was obviously different from DCW and $\mathrm{PCL}$. In the N1 group, the N1P1 remained unchanged, and the NIP3 group continued to increase. In addition, the N1P2 continued to increase, while the curve was higher than the normal group. This indicated that when the concentration of $\mathrm{N}$ was appropriate, the medium concentration of $\mathrm{P}$ was more suitable for the accumulation of phycocyanin.

3.1.2. Research on Optimal Environment Conditions. On the 18th day, the average response value of DCW, PCL, and PCD of 9 experimental groups was analyzed by SPSS (Table 2). For the average response values of DCW, PCL, and $\mathrm{PCD}$, the range of $\mathrm{N}(0.284,58.858$, and 96.093$)$ was greater 
TABLE 2: The response value of dry cell weight (DCW), phycocyanin content in liquid (PCL), and phycocyanin content in dried Spirulina $(\mathrm{PCD})$ in different culture conditions.

\begin{tabular}{lcccccccc}
\hline & $\mathrm{N} 1$ & $\mathrm{~N} 2$ & $\mathrm{~N} 3$ & $R_{\mathrm{N}}$ & $\mathrm{P} 1$ & $\mathrm{P} 2$ & $R_{\mathrm{P}}$ \\
\hline DCW & 0.332 & 0.130 & 0.048 & 0.284 & 0.147 & 0.168 & 0.195 \\
PCL & 63.643 & 17.081 & 4.785 & 58.858 & 20.962 & 29.580 & 34.966 \\
PCD & 190.997 & 126.881 & 94.094 & 96.093 & 113.588 & 141.300 & 157.084 & 14.004 \\
\hline
\end{tabular}

Each content was measured once. N1, N2, and N3 were different concentrations of $\mathrm{NH}_{4} \mathrm{Cl}(0.1,0.2$, and $0.3 \mathrm{mg} / \mathrm{L})$; $\mathrm{P} 1, \mathrm{P} 2$, and $\mathrm{P} 3$ were different concentrations of $\mathrm{K}_{2} \mathrm{HPO}_{4}(0.02,0.42$, and $0.82 \mathrm{mg} / \mathrm{L}) ; R_{\mathrm{N}}$ and $R_{\mathrm{P}}$ were the range of response value caused by different concentrations of $\mathrm{N}$ and $\mathrm{P}$.

TABLE 3: Variance analysis of dry cell weight (DCW), phycocyanin content in liquid (PCL), and phycocyanin content in dried Spirulina (PCD).

\begin{tabular}{|c|c|c|c|c|c|c|}
\hline & Source & Type III sum of squares & $\mathrm{D} f$ & Mean square & $\mathrm{F}$ & $\mathrm{P}$ \\
\hline \multirow{2}{*}{ DCW } & $\mathrm{N}$ & 0.128 & 2 & 0.064 & 957.125 & 0.000 \\
\hline & $\mathrm{P}$ & 0.004 & 2 & 0.002 & 26.375 & 0.005 \\
\hline \multirow{2}{*}{ PCL } & $\mathrm{N}$ & 5783.498 & 2 & 2891.749 & 113.351 & 0.000 \\
\hline & $\mathrm{P}$ & 299.361 & 2 & 149.680 & 5.867 & 0.065 \\
\hline \multirow{2}{*}{ PCD } & $\mathrm{N}$ & 14575.954 & 2 & 7287.977 & 63.565 & 0.001 \\
\hline & $\mathrm{P}$ & 2908.944 & 2 & 1454.472 & 12.686 & 0.019 \\
\hline
\end{tabular}

than the range of $\mathrm{P}(0.048,14.004$, and 43.496), which indicated that the $\mathrm{N}$ had a greater impact on the growth of Spirulina. In order to further analyze the extent of the effects of $\mathrm{N}$ and $\mathrm{P}$, we conducted an analysis of variance (Table 3 ). The influence of $\mathrm{N}$ on DCW $(p<0.001)$, PCL $(p<0.001)$, and $\operatorname{PCD}(p=0.001)$ was very significant, and all were greater than $\mathrm{P}$. Therefore, it can be considered that the $\mathrm{N}$ was the main factor and the $\mathrm{P}$ was the secondary factor in this experiment. According to the average of the response values in Table 3, N1P3 was the best cultivation conditions. However, the analysis of variance found that the influence of $\mathrm{P}(p=0.065)$ on PCL was not very significant, and multiple comparison analysis was conducted (Table S1). The results showed that P2 and P3 have little difference between the experimental results. In Section 3.1.1, we had found that the medium concentration of $\mathrm{P}$ was more suitable for the growth of Spirulina when the concentration of $\mathrm{N}$ was appropriate. Therefore, the optimal condition was finally determined as $\mathrm{N} 1 \mathrm{P} 2\left(\mathrm{NH}_{4} \mathrm{Cl} 0.1 \mathrm{~g} / \mathrm{L}\right.$ and $\left.\mathrm{K}_{2} \mathrm{HPO}_{4} 0.42 \mathrm{~g} / \mathrm{L}\right)$.

3.2. Research on Rapid Detection of Phycocyanin Based on Raman Spectroscopy. As the phycocyanin market gradually expanded, more convenient and efficient monitoring methods will be required during the production process. This study was the first in situ detection of phycocyanin using a portable Raman spectrometer. The Spirulina was cultivated under the optimal culture conditions obtained in Section 3.1. A portable Raman spectrometer was used to collect information on Spirulina fluid and combined with LSSVM to establish a PCL concentration prediction model. A total of 96 spectral data were obtained. There were fluctuations in $151-935 \mathrm{~cm}^{-1}$ and $1720-3000 \mathrm{~cm}^{-1}$ (Figure S1), resulting in a low signal-to-noise ratio. Therefore, we select $935-1720 \mathrm{~cm}^{-1}$ (Figure 5(a)) for subsequent analysis. The Raman spectra show that there are 7 characteristic absorption peaks for these Spirulina fluid samples. Table 4 summarizes the assignment of these characteristic peaks [25-29].
In order to explain mechanism behind the formation of these absorption peaks, quantum chemistry calculations of the phycocyanin molecules were carried out with theoretical value calculated by DFT. The molecular vibration modes of each peak were determined by comparing the experimental and theoretical spectra. As shown in Figure 5(b), the characteristic peaks that were measured at 1272, 1337, and $1432 \mathrm{~cm}^{-1}$ and which attributed to protein could be matched with the simulated peaks at 1276, 1340, and $1431 \mathrm{~cm}^{-1}$, respectively. It can be considered that these three peaks represented phycocyanin in the data measured by Raman spectrometer.

To further determine the relationship between the concentration of PCL and the intensity of characteristic peak, five different PCL concentrations were collected, and the corresponding average Raman spectroscopy is shown in Figure 6(a). The intensity decreased gradually with the decrease in PCL concentration at the characteristic peaks that attributed to phycocyanin $\left(1272,1337\right.$, and $\left.1432 \mathrm{~cm}^{-1}\right)$. The linear regression models between these three characteristic peaks and the PCL concentration were established. As shown in Figures 6(c)-6(e), the intensity of 1272, 1337, and $1432 \mathrm{~cm}^{-1}$ had correlation coefficients $\left(R^{2}\right)$ of 0.789 , 0.811 , and 0.800 , respectively. We further establish linear regression model between the sum of the three peak intensities and the PCL concentration. As shown in Figure 6(b), the correlation coefficient $\left(R^{2}\right)$ was significantly increased to 0.89 , eliminating the influence of the error of a single characteristic peak on the results.

It was worth noting in Figure 6(a) that the intensity of 1152 and $1518 \mathrm{~cm}^{-1}$ attributed to $\beta$-carotene also decreased gradually with the decrease in PCL concentration. This indicated that the intensity of all the seven characteristic peaks had relationship with the concentration of PCL. Then, we implemented a simple correlation analysis between each peak and their response to PCL concentration (Figure 7(a)). Firstly, we can find that Pearson's correlation coefficients of the seven characteristic peaks with PCL concentration were 


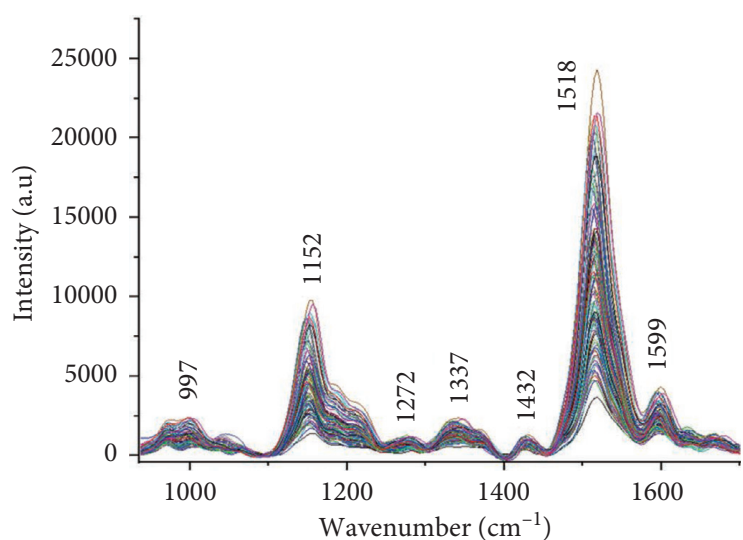

(a)

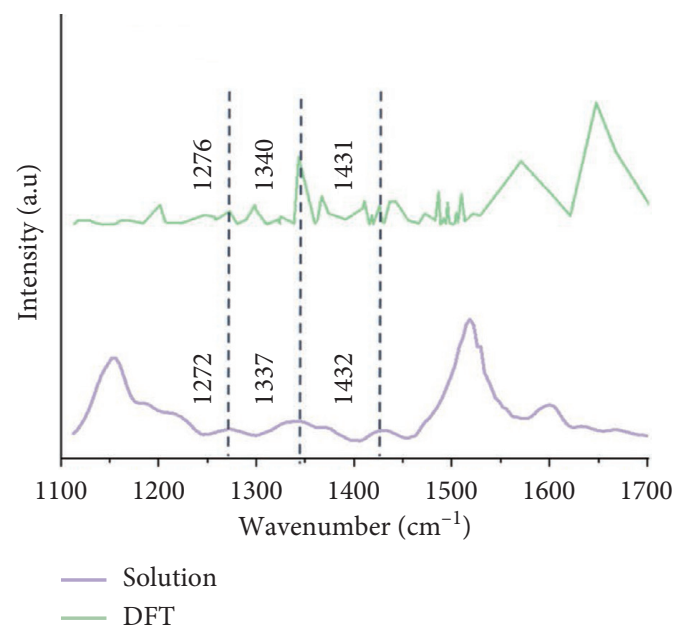

(b)

Figure 5: (a) Spectrum of Spirulina fluid after processing; (b) comparison of the theory calculation of phycocyanin by density functional theory and the spectrum of Spirulina fluid detected by Raman spectrometer system.

TABLE 4: The assignment of characteristic Raman peaks of Spirulina.

\begin{tabular}{lcc}
\hline Raman shift $\left(\mathrm{cm}^{-1}\right)$ & Assignment & Analytes \\
\hline 997 & $\delta($ ring $)$ & Phenylalanine \\
1152 & $\delta(\mathrm{CH})$ & $\beta$-Carotene \\
1272 & Amide III, $v(\mathrm{C}-\mathrm{N})$ & $\beta$-Carotene \\
1337 & $\delta\left(\mathrm{CH}_{3}\right), \delta(\mathrm{CH})$ & Proteins \\
1432 & $\mathrm{~T}\left(\mathrm{CH}_{3} \mathrm{CH}_{2}\right), \omega\left(\mathrm{CH}_{3} \mathrm{CH}_{2}\right)$ & Proteins, lipids \\
1518 & $\delta\left(\mathrm{CH}_{2}\right)$ & $\beta$-Carotene \\
1599 & $\mathrm{C}=\mathrm{C}$ stretching & $\beta$-Carotene \\
\hline
\end{tabular}

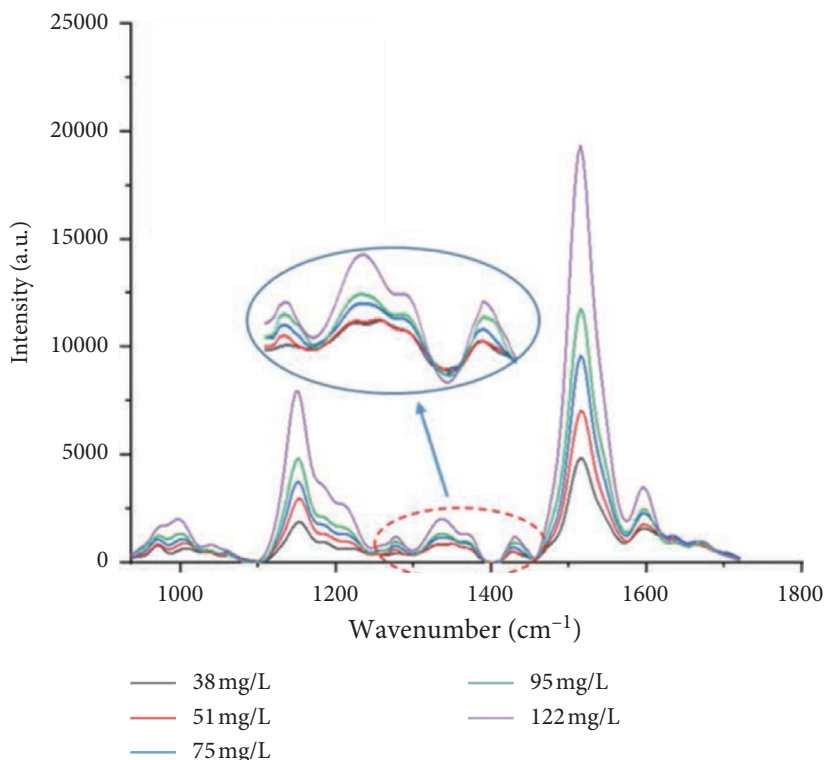

(a)

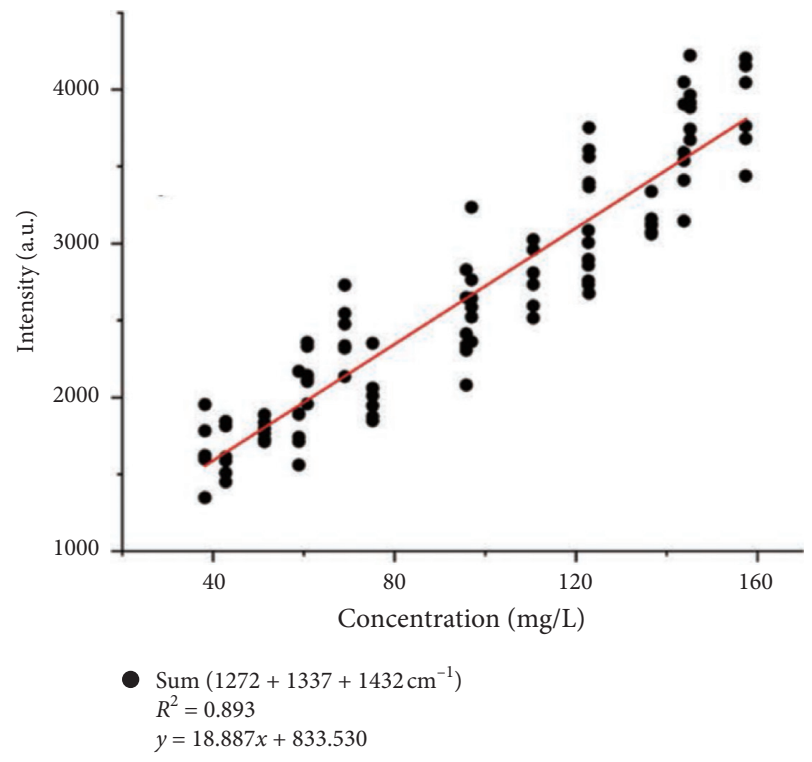

(b)

Figure 6: Continued. 


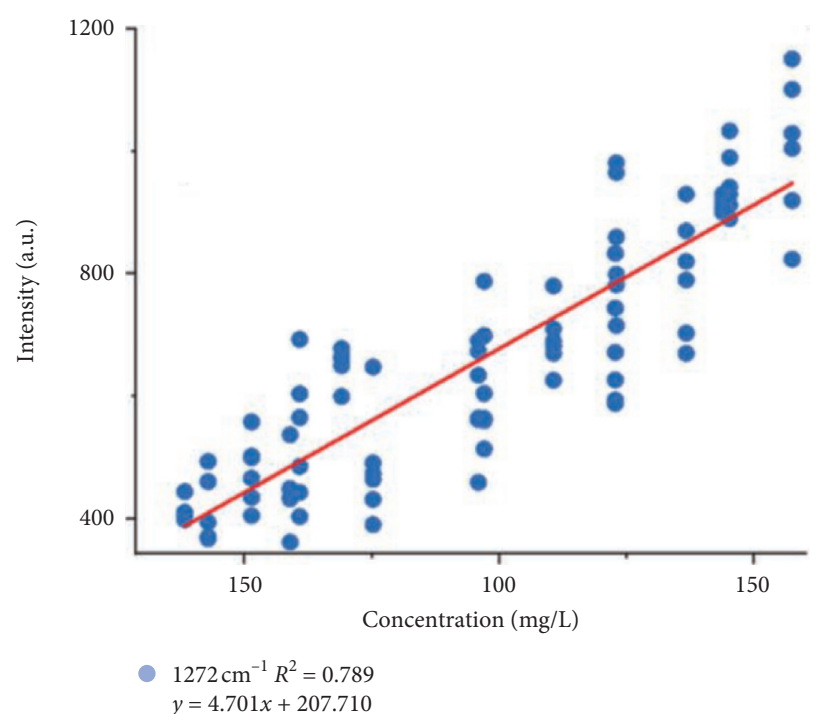

(c)

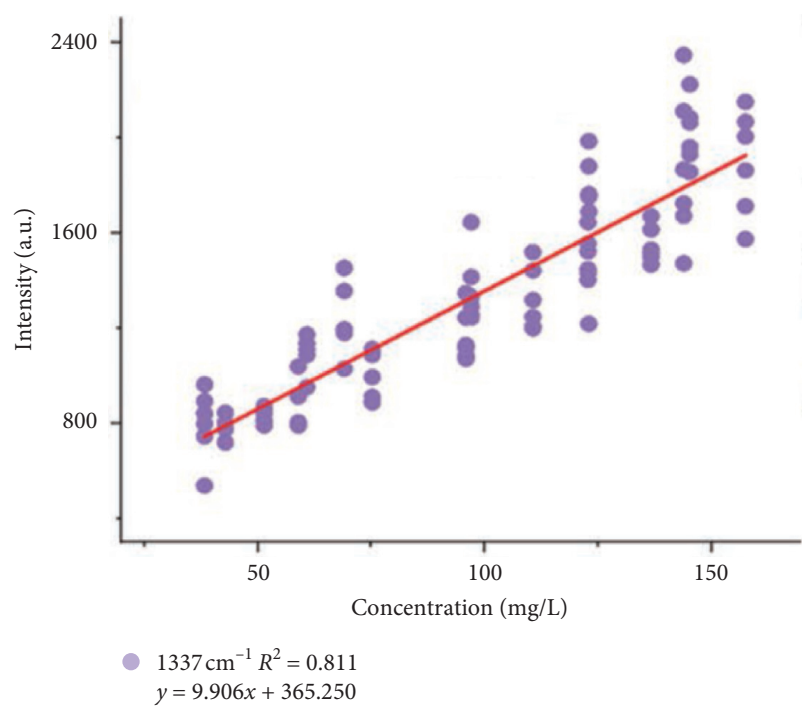

(d)

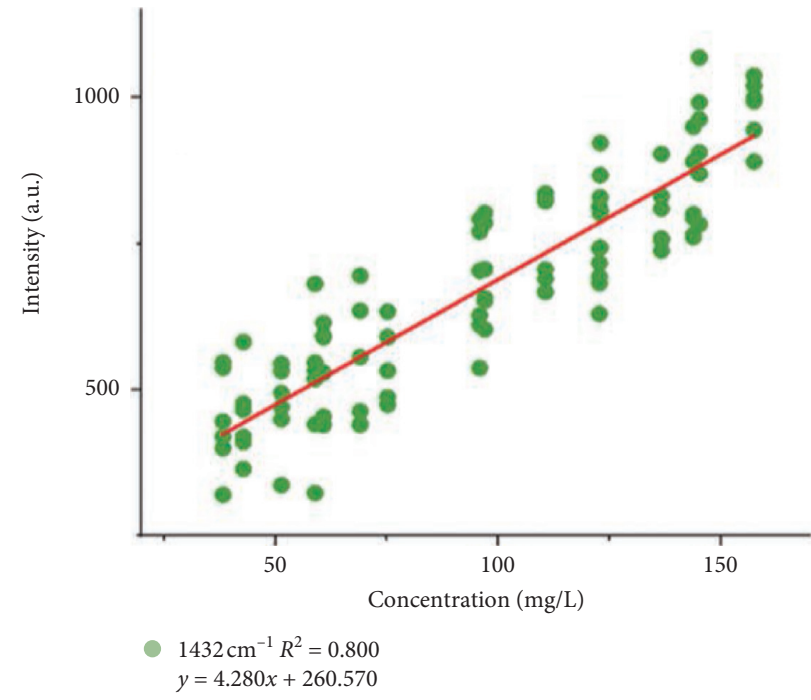

(e)

Figure 6: (a) The Raman spectra of five different concentrations of PCL in Spirulina; (b) the linear regression equation between the sum of the three peak $\left(1272 \mathrm{~cm}^{-1}, 1337 \mathrm{~cm}^{-1}\right.$, and $\left.1432 \mathrm{~cm}^{-1}\right)$ intensities and the PCL concentration; the linear regression equations between Raman peak intensity and PCL concentration at different Raman peaks: (c) $1272 \mathrm{~cm}^{-1}$; (d) $1337 \mathrm{~cm}^{-1}$; (e) $1432 \mathrm{~cm}^{-1}$.

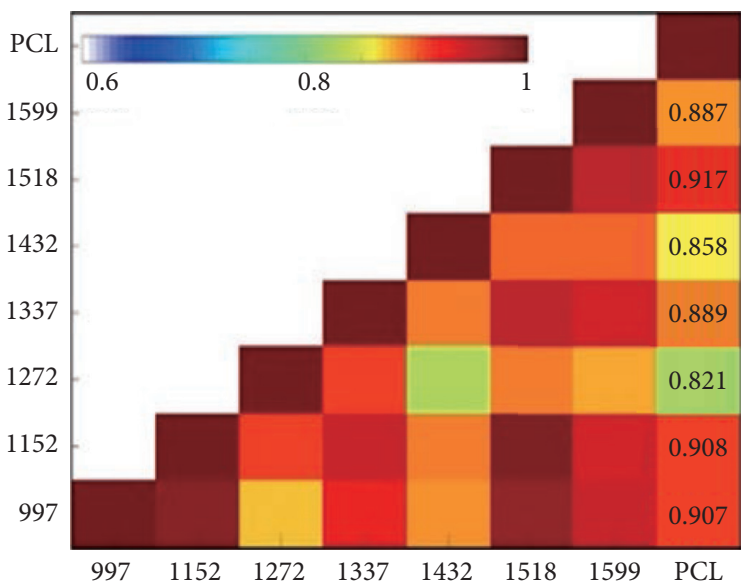

(a)

Figure 7: Continued. 


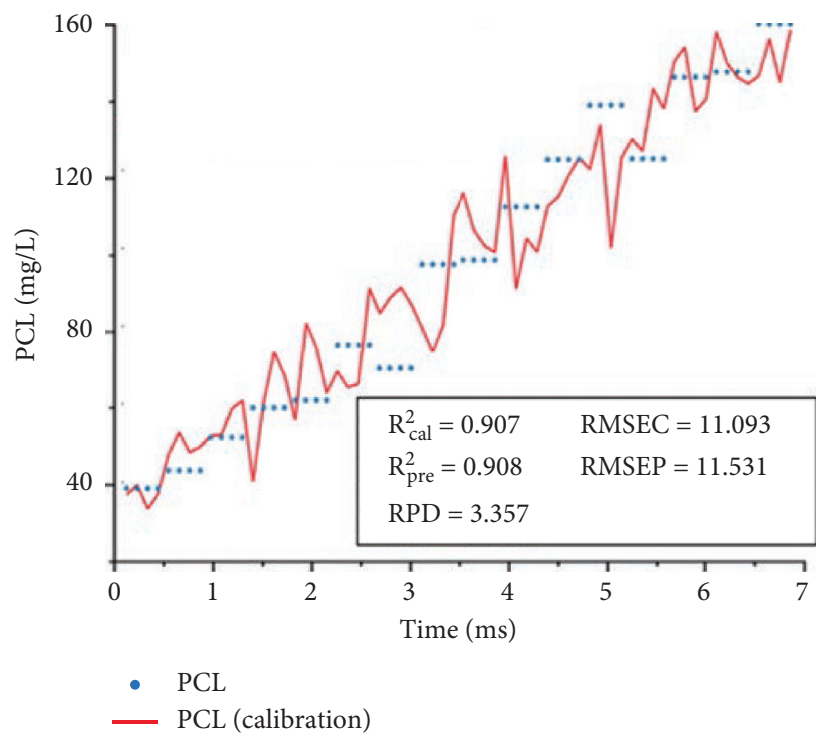

(b)

FiguRE 7: (a) Correlation coefficients between each characteristic wavenumber and their response to the content of phycocyanin content in liquid (PCL); (b) least squares support vector machine (LSSVM) modeling process of characteristic peaks detected by the $405 \mathrm{~nm}$ portable Raman spectrometer system.

higher than 0.8 . Then, the peaks that attributed to $\beta$-carotene displayed strong correlations with the characteristic peaks of phycocyanin. The $1518 \mathrm{~cm}^{-1}$ displayed the strongest correlation with the PCL, while it had no correspondence with phycocyanin. $\beta$-Carotene is part of the pigment-protein complex in microalgae and plays an important role in the energy transfer process of photosynthesis. It can absorb the strongest light in the blue region (400-500 nm) and transfer the excitation energy to chlorophyll, making photosynthesis effective in a wider wavelength range. Therefore, $\beta$-carotene can reflect the growth of Spirulina. The healthier the Spirulina is, the more beneficial it is to the synthesis of phycocyanin. Therefore, all seven characteristic peaks are helpful for the study of PCL concentration.

LSSVM model was finally implemented to evaluate the actual roles of these seven Raman characteristic peaks. The optimized $\gamma$ and $\delta^{2}$ of LSSVM were set to 11.294 and 42.39, respectively. The process and result are shown in Figure $7(\mathrm{~b})$. The overall $R^{2}$ was higher than 0.9 . The $R_{\text {pre }}^{2}$ was 0.908 , RMSEP was 11.531, and RPD was higher than 3 , which indicated the model was successful [30]. Therefore, the modeling results indicated that the rapid detection of phycocyanin by the $405 \mathrm{~nm}$ portable Raman spectrometer was feasible.

\section{Conclusions}

Overall, we demonstrated that appropriate concentration of $\mathrm{N}$ and $\mathrm{P}$ is very important for the accumulation of phycocyanin in the cultivation process of Spirulina. When the high concentration of $\mathrm{N}$ causes significant damage to Spirulina, the high concentration of $\mathrm{P}$ may have a certain protective effect on the growth of Spirulina. When the concentration of $\mathrm{N}$ is appropriate, the medium concentration of $\mathrm{P}$ has a better effect on the synthesis of phycocyanin. The study obtained the most suitable conditions: $\mathrm{NH}_{4} \mathrm{Cl}, 0.1 \mathrm{~g} / \mathrm{L}$, and $\mathrm{K}_{2} \mathrm{HPO}_{4}, 0.42 \mathrm{~g} / \mathrm{L}$. We also detected the Spirulina by portable Raman spectrometer and obtained the characteristic peaks of phycocyanin $\left(1272,1337\right.$, and $\left.1432 \mathrm{~cm}^{-1}\right)$. There was a good linear correlation between the sum of the peak intensities and the PCL concentration $(y=18.887 x+833.530$, $\left.R^{2}=0.890\right)$. The LSSVM model based on the characteristic peaks obtained good result, the $R_{\text {pre }}^{2}$ was 0.907 , and the RPD was 3.357. This study provides a method for realtime monitoring of phycocyanin content in Spirulina, which is of great significance for formulating dynamic cultivation strategies.

\section{Data Availability}

The data used to support the findings of this study are included within the article.

\section{Conflicts of Interest}

The authors declare that there are no conflicts of interest regarding the publication of this paper.

\section{Acknowledgments}

This research was financially supported by the Key Projects of International Scientific and Technological Innovation Cooperation among Governments under National Key R\&D Plan (Grant no. 2019YFE0103800) and National Natural Science Foundation of China (Grant no. 31402318). 


\section{Supplementary Materials}

The Supplementary Materials can be found online. Figure S1: raw spectrum of Spirulina fluid detected by $405 \mathrm{~nm}$ portable Raman spectrometer system; Table S1:multiple comparative analysis of the effect of different concentrations of P on PCL. (Supplementary Materials)

\section{References}

[1] G.-C. J. Antonio, M.-A. Francisco, A.-F. F. Gabriel, and M.-G. Emilio, "Microalgae research worldwide," Algal Research, vol. 35, pp. 50-60, 2018.

[2] F. Gao, C. Li, Z.-H. Yang et al., "Continuous microalgae cultivation in aquaculture wastewater by a membrane photobioreactor for biomass production and nutrients removal," Ecological Engineering, vol. 92, pp. 55-61, 2016.

[3] D. Zeng, B. Chen, X.-L. Hu et al., "Research progress of microalgae cultivation using wastewaters," Genomics and Applied Biology, vol. 38, pp. 3105-3111, 2019.

[4] R. Chaiklahan, N. Chirasuwan, V. Loha et al., "Stepwise extraction of high-value chemicals from Arthrospira (Spirulina) and an economic feasibility study," Biotechnology Reports (Amsterdam, Netherlands), vol. 20, Article ID e00280, 2018.

[5] S. Sekar and M. Chandramohan, "Phycobiliproteins as a commodity: trends in applied research, patents and commercialization," Journal Of Applied Phycology, vol. 20, no. 2, pp. 113-136, 2008.

[6] S. Wang, Y. Meng, J. Liu, X. Cao, and S. Xue, "Accurate quantification of astaxanthin from Haematococcus pluvialis using DMSO extraction and lipase-catalyzed hydrolysis pretreatment," Algal Research, vol. 35, pp. 427-431, 2018.

[7] F.-F. Chu, P.-N. Chu, P.-J. Cai, W.-W. Li, P. K. S. Lam, and R. J. Zeng, "Phosphorus plays an important role in enhancing biodiesel productivity of Chlorella vulgaris under nitrogen deficiency," Bioresource Technology, vol. 134, pp. 341-346, 2013.

[8] M. Isleten-Hosoglu, I. Gultepe, and M. Elibol, "Optimization of carbon and nitrogen sources for biomass and lipid production by Chlorella saccharophila under heterotrophic conditions and development of Nile red fluorescence based method for quantification of its neutral lipid content," Biochemical Engineering Journal, vol. 61, pp. 11-19, 2012.

[9] M. Sobiechowska-Sasim, J. Stoń-Egiert, and A. Kosakowska, "Quantitative analysis of extracted phycobilin pigments in cyanobacteria-an assessment of spectrophotometric and spectrofluorometric methods," Journal Of Applied Phycology, vol. 26, no. 5, pp. 2065-2074, 2014.

[10] A. Kaczor, K. Turnau, and M. Baranska, "In situ Raman imaging of astaxanthin in a single microalgal cell," The Analyst, vol. 136, no. 6, pp. 1109-1102, 2011.

[11] K. Li, J. Cheng, Q. Ye et al., "In vivo kinetics of lipids and astaxanthin evolution in Haematococcus pluvialis mutant under $15 \% \mathrm{CO}_{2}$ using Raman microspectroscopy," Bioresource Technology, vol. 244, pp. 1439-1444, 2017.

[12] Š. Moudř́ḱková, P. Mojzeš, V. Zachleder, C. Pfaff, and D. Behrendt, "Raman and fluorescence microscopy sensing energy-transducing and energy-storing structures in microalgae," Algal Research, vol. 16, pp. 224-232, 2016.

[13] M. Hosokawa, M. Ando, S. Mukai et al., "In vivo live cell imaging for the quantitative monitoring of lipids by using Raman microspectroscopy," Analytical Chemistry, vol. 86, no. 16, pp. 8224-8230, 2014.
[14] O. Samek, A. Jonáš, Z. Pilát et al., "Raman microspectroscopy of individual algal cells: sensing unsaturation of storage lipids in vivo," Sensors, vol. 10, no. 9, pp. 8635-8651, 2010.

[15] L.-d. Chiu, S.-H. Ho, R. Shimada, N.-Q. Ren, and T. Ozawa, "Rapid in vivo lipid/carbohydrate quantification of single microalgal cell by Raman spectral imaging to reveal salinityinduced starch-to-lipid shift," Biotechnology for Biofuels, vol. 10, 2017.

[16] W. Grudzinski, I. Krzeminska, R. Luchowski, A. Nosalewicz, and W. I. Gruszecki, "Strong-light-induced yellowing of green microalgae Chlorella: a study on molecular mechanisms of the acclimation response," Algal Research, vol. 16, pp. 245-254, 2016.

[17] J. Jehlicka and A. Oren, "Use of a handheld Raman spectrometer for fast screening of microbial pigments in cultures of halophilic microorganisms and in microbial communities in hypersaline environments in nature," Journal of Raman Spectroscopy, vol. 44, pp. 1285-1291, 2013.

[18] M. Ando, M. Sugiura, H. Hayashi, and H.-o. Hamaguchi, "1064 nm deep near-infrared (NIR) excited Raman microspectroscopy for studying photolabile organisms," Applied Spectroscopy, vol. 65, no. 5, pp. 488-492, 2011.

[19] Y. Shao, H. Fang, H. Zhou, Q. Wang, Y. Zhu, and Y. He, "Detection and imaging of lipids of Scenedesmus obliquus based on confocal Raman microspectroscopy," Biotechnology for Biofuels, vol. 10, 2017.

[20] P. Spolaore, C. Joannis-Cassan, E. Duran, and A. Isambert, "Commercial applications of microalgae," Journal Of Bioscience And Bioengineering, vol. 101, no. 2, pp. 87-96, 2006.

[21] K. R. Ramya and A. Venkatnathan, "Stability and reactivity of methane clathrate hydrates: insights from density functional theory," The Journal of Physical Chemistry A, vol. 116, no. 29, pp. 7742-7745, 2012.

[22] D. Wu, Y. He, S. Feng, and D.-W. Sun, "Study on infrared spectroscopy technique for fast measurement of protein content in milk powder based on LS-SVM," Journal Of Food Engineering, vol. 84, no. 1, pp. 124-131, 2008.

[23] M. C. Damiani, C. A. Popovich, D. Constenla, and P. I. Leonardi, "Lipid analysis in Haematococcus pluvialis to assess its potential use as a biodiesel feedstock," Bioresource Technology, vol. 101, no. 11, pp. 3801-3807, 2010.

[24] Y. Cao, Z. Li, X. Hu et al., "Effects of phosphorus concentration and nitrogen-phosphorus ratio on absorption of nitrogen and phosphorus by Chlorella pyrenoidosa," Ecologic Science, vol. 36, pp. 34-40, 2017.

[25] X.-L. Li and Y. He, "Determination of carotenoids contents in tea leaves based on rman spectroscopy," Spectroscopy And Spectral Analysis, vol. 37, pp. 3465-3470, 2017.

[26] J. Ge, L. Sun, X.-J. Shen, Y.-F. Sha, T.-X. Huang, and Y.-P. Du, "Simultaneous detection of lutein and $\beta$-carotene in tobacco by using Raman spectroscopy combined with partial least squares," Spectroscopy And Spectral Analysis, vol. 39, pp. 3519-3524, 2019.

[27] S. Schlücker, A. Szeghalmi, M. Schmitt, J. Popp, and W. Kiefer, "Density functional and vibrational spectroscopic analysis of $\beta$-carotene," Journal of Raman Spectroscopy, vol. 34, pp. 413-419, 2003.

[28] W. Claudia, U. S. Hubert, R. Clemens et al., “Assessing various Infrared (IR) microscopic imaging techniques for postmortem interval evaluation of human skeletal remains," PLoS One, vol. 12, Article ID e0174552, 2017.

[29] G. Das, F. Mecarini, F. Gentile et al., "Nano-patterned SERS substrate: application for protein analysis vs. temperature," 
Biosensors and Bioelectronics, vol. 24, no. 6, pp. 1693-1699, 2009.

[30] R. K. Saini and Y.-S. Keum, "Progress in microbial carotenoids production," Indian Journal Of Microbiology, vol. 57, no. 1, pp. 129-130, 2017. 